\title{
SPERM TRANSPORT IN THE FEMALE RABBIT: THE EFFECT OF INSEMINATE VOLUME AND SPERM DENSITY
}

\author{
D. B. MORTON* AND T. D. GLOVER \\ Unit of Reproductive Biology, University of Liverpool, \\ Life Sciences Building, Crown Street, Liverpool L69 3BX
}

(Received 19th September 1973)

\begin{abstract}
Summary. Ovariectomized oestrogen-treated rabbits were inseminated with semen of variable sperm density and volume. Does were killed $10 \mathrm{hr}$ after insemination and the number of spermatozoa in the vagina, cervix, uterus and oviduct was determined. It was found that sperm counts from all regions of the genital tract were directly related to the total number of spermatozoa in the inseminate. When the number of spermatozoa in the inseminate was held constant, more spermatozoa were recovered from the cervix and uterus with the smaller inseminate volumes. All regional counts were positively correlated with one another. Proportionately fewer spermatozoa were recovered from the genital tract when the inseminate contained larger sperm numbers.
\end{abstract}

\section{INTRODUGTION}

In rabbits, it has been shown that between $10^{5}$ and $10^{6}$ spermatozoa only are needed to ensure maximum fertilization (Walton, 1927; Rowlands, 1944; Chang, 1946; Austin, 1948; Wales, Martin \& O'Shea, 1965). The gross dilution of semen necessary to obtain these small numbers of spermatozoa might be expected to interfere with sperm function, thereby affecting fertilization. Experiments have shown, however, that reduced fertility can be primarily explained by small numbers of spermatozoa in the inseminate and, to a minor extent, by the parity of the doe (multiparous does showed poorer fertility than virgin does when inseminated with low density semen) but that inseminate volume did not appear to be important (Wales et al., 1965). Whether the drop in fertility with small sperm numbers is due to failure of sperm transport, poor sperm survival, the inability of spermatozoa to fertilize the egg or to early embryonic death is not known.

The present study was concerned with measuring the distribution of spermatozoa in does which had been inseminated with semen that varied both in volume and sperm number. It was hoped to establish whether the number of spermatozoa in the cervical reservoir and the oviduct was significantly affected by the sperm density and volume of the inseminate, and whether, therefore,

* Present address: Strangeways' Research Laboratory, Wort's Causeway, Gambridge CB1 4RN. 
sperm transport was the cause of the reduced fertilization rate found when does are inseminated with small numbers of spermatozoa.

\section{MATERIALS AND METHODS}

In an attempt to minimize biological variation, the hormonal status of the does was controlled by ovariectomy and oestrogen treatment and only sexually mature virgin does weighing between 3 and $4 \mathrm{~kg}$ were used. Forty-six New Zealand White does were ovariectomized and 4 to 6 weeks later were injected intramuscularly with $1 \mu \mathrm{g}$ oestradiol benzoate ('Oestroform', B.D.H. Ltd) in $0.5 \mathrm{ml}$ olive oil each day, for 4 days, a dosage rate that has been shown to be of physiological order (Noyes, Adams \& Walton, 1959). The animals were grouped in a basic ' 2 factorial' design of four inseminate volumes $(0 \cdot 1,0 \cdot 3$, 1.0 and $2.0 \mathrm{ml})$ against four sperm numbers $\left(0.5,1.0,50.0\right.$ and $\left.500.0 \times 10^{6}\right)$, resulting in sixteen separate groups.

Semen samples of good motility were pooled and the requisite concentration of spermatozoa was obtained within $1 \mathrm{hr}$ of collection, either by diluting with saline $(0.9 \% \mathrm{NaCl})$ or by centrifugation $(1500 \mathrm{~g}$ for $2 \mathrm{~min}$ ) followed by dilution with saline. Intravaginal insemination was carried out using a conventional glass inseminating pipette through which a fine bore polythene tube was threaded. The polythene tube was connected to a syringe filled with the appropriate volume of semen. Animals were killed $24 \mathrm{hr}$ after the fourth oestrogen injection and insemination was timed to be 10 to $11 \mathrm{hr}$ before death, i.e. a time interval equivalent to that between mating and ovulation in the intact rabbit. The number of spermatozoa present in the oviduct, uterus, cervix and vagina was estimated from flushings of the tract (see Morton, 1970; Morton \& Glover, 1974). Statistical analyses were worked out using the logarithm $(x+1)$ of the number of spermatozoa counted, as this conversion normalized the data and reduced artificial weightings caused by exceptionally large counts in any one group.

\section{RESULTS}

The overall mean number of spermatozoa recovered from each region of the female tract is shown in Table 1: the data have been pooled from the sixteen groups to give overall means for either a given inseminate volume or sperm number. Table 2 summarizes the analysis of variance for the data and it can be seen that most of the variation between groups may be attributed to the effect of sperm number. The volume of the inseminate had a significant effect on sperm numbers in the cervix and uterus but not on those in the vagina or oviduct; smaller inseminate volumes significantly raised cervical and uterine counts. No significant difference was found in any region between left and right sides of the tract. The highly significant interaction between inseminate volume and the cervical sperm count was probably caused by an exceptionally small cervical count in two out of three rabbits in the group inseminated with $50 \times 10^{6}$ spermatozoa in $0.1 \mathrm{ml}$ : this resulted in larger volumes having a relatively beneficial effect, contrary to results in the other groups. Similar exceptional 
Table 1. The mean number of spermatozoa recovered from each region of the female genital tract of does inseminated with various inseminate volumes and various sperm numbers

\begin{tabular}{|c|c|c|c|c|}
\hline & Vagina & Cervix & Uterus & Oviduct \\
\hline $\begin{array}{c}\text { Number of spermatozoa in } \\
\text { the inseminate }\left(\times 10^{6}\right) \\
0.5\end{array}$ & $\begin{array}{c}21,000 \\
(106,022)\end{array}$ & $\begin{array}{c}15,100 \\
(17,786)\end{array}$ & $\begin{array}{c}2,240 \\
(5,199)\end{array}$ & $\begin{array}{c}43 \\
(412)\end{array}$ \\
\hline $1 \cdot 0$ & $\begin{array}{c}26,900 \\
(157,860)\end{array}$ & $\begin{array}{c}11,200 \\
(25,348)\end{array}$ & $\begin{array}{c}2,400 \\
(4,983)\end{array}$ & $\begin{array}{c}33 \\
(176)\end{array}$ \\
\hline $50 \cdot 0$ & $\begin{array}{c}759,000 \\
(1,232,413)\end{array}$ & $\begin{array}{c}398,000 \\
(645,130)\end{array}$ & $\begin{array}{c}93,300 \\
(214,488)\end{array}$ & $\begin{array}{c}398 \\
(1930)\end{array}$ \\
\hline $500 \cdot 0$ & $\begin{array}{c}4,470,000 \\
(5,414,088)\end{array}$ & $\begin{array}{c}1,200,000 \\
(1,429,412)\end{array}$ & $\begin{array}{c}269,000 \\
(572,111)\end{array}$ & $\begin{array}{l}1510 \\
(2936)\end{array}$ \\
\hline $\begin{array}{l}\text { Volume of inseminate (ml) } \\
0.1\end{array}$ & $\begin{array}{c}363,000 \\
(1,319,243)\end{array}$ & $\begin{array}{c}120,000 \\
(391,139)\end{array}$ & $\begin{array}{c}17,400 \\
(79,901)\end{array}$ & $\begin{array}{c}174 \\
(630)\end{array}$ \\
\hline $0 \cdot 3$ & $\begin{array}{c}219,000 \\
(3,287,966)\end{array}$ & $\begin{array}{c}129,000 \\
(700,600)\end{array}$ & $\begin{array}{c}27,600 \\
(338,929)\end{array}$ & $\begin{array}{c}234 \\
(1001)\end{array}$ \\
\hline 1.0 & $\begin{array}{c}129,000 \\
(3,589,000)\end{array}$ & $\begin{array}{c}60,300 \\
(477,584)\end{array}$ & $\begin{array}{c}14,800 \\
(108,225)\end{array}$ & $\begin{array}{c}155 \\
(951)\end{array}$ \\
\hline $2 \cdot 0$ & $\begin{array}{c}195,000 \\
(1,944,175)\end{array}$ & $\begin{array}{c}87,100 \\
(548,354)\end{array}$ & $\begin{array}{c}19,100 \\
(269,728)\end{array}$ & $\begin{array}{c}132 \\
(2871)\end{array}$ \\
\hline
\end{tabular}

Mean values are in two forms: the anti-log of the logarithmic mean with the arithmetic mean in parentheses.

instances could be found to account for the other two interactions in the vagina and oviduct, though these interactions were only significant at the $5 \%$ level. The considerable biological variation encountered in work of this kind is illustrated by the large coefficients of variation (Table 2) and the marked differences between the arithmetic and logarithmic means (Table 1).

The effects of sperm number and volume of the inseminate are further illustrated in Text-fig. 1. Data have been pooled to consider only volume or sperm number for each of the sixteen groups of rabbits and least significant

Table 2. Analysis of variance showing the relationships between qualities of the inseminate and regional sperm counts

\begin{tabular}{|c|c|c|c|c|c|c|c|c|}
\hline \multirow[b]{2}{*}{ Source of variation } & \multicolumn{8}{|c|}{ Region of the tract } \\
\hline & d.f. & $\underset{F}{\text { Vagina }}$ & d.f. & $\underset{\mathrm{F}}{\text { Cervix }}$ & $d . f$ & $\begin{array}{l}\text { Uterus } \\
\mathrm{F}\end{array}$ & d.f. & $\begin{array}{c}\text { Oviduct } \\
\mathrm{F}\end{array}$ \\
\hline $\begin{array}{l}\text { Inseminate volume } \\
\text { Sperm number }\end{array}$ & & $\begin{array}{c}1 \cdot 69 \\
38 \cdot 26^{* * *}\end{array}$ & & $\begin{array}{c}7 \cdot 25^{* *} \\
207 \cdot 58^{* * *}\end{array}$ & $\begin{array}{l}3 \\
3\end{array}$ & $\begin{array}{c}4 \cdot 48^{* *} \\
85 \cdot 94 * * *\end{array}$ & $\begin{array}{l}3 \\
3\end{array}$ & $\begin{array}{l}1 \cdot 10 \\
22 \cdot 90 * * *\end{array}$ \\
\hline $\begin{array}{l}\text { sperm no.) } \\
\text { Side of tract } \\
\text { Error }\end{array}$ & $\begin{array}{r}9 \\
27\end{array}$ & $2 \cdot 29$ & $\begin{array}{r}9 \\
1 \\
75\end{array}$ & $\begin{array}{l}5 \cdot 46^{* * *} \\
0 \cdot 33\end{array}$ & $\begin{array}{r}9 \\
1 \\
75\end{array}$ & $\begin{array}{l}1.52 \\
0.61\end{array}$ & $\begin{array}{r}9 \\
1 \\
74\end{array}$ & $\begin{array}{l}2 \cdot 37 * \\
0 \cdot 59\end{array}$ \\
\hline$(\%)$ & & $11 \cdot 62$ & & 6.92 & & 12.87 & & $36 \cdot 44$ \\
\hline
\end{tabular}

Flushings from the vagina of three rabbits and the oviduct of one rabbit are not included.

$* P<0.05 ;{ }^{* *} P<0.01 ; * * * P<0.001$. 
differences have been calculated between these overall means. Inseminate volume was found to have no significant effect on sperm counts in the vagina, uterus and oviduct (Text-fig. la), but a significant effect of volume was noted for the cervix; more spermatozoa were recovered from the cervix with smaller inseminate volumes than with larger volumes (see also Table 1). When sperm number was considered, a positive correlation was found between the number of spermatozoa in the inseminate and the regional counts (Text-fig. 1b). There
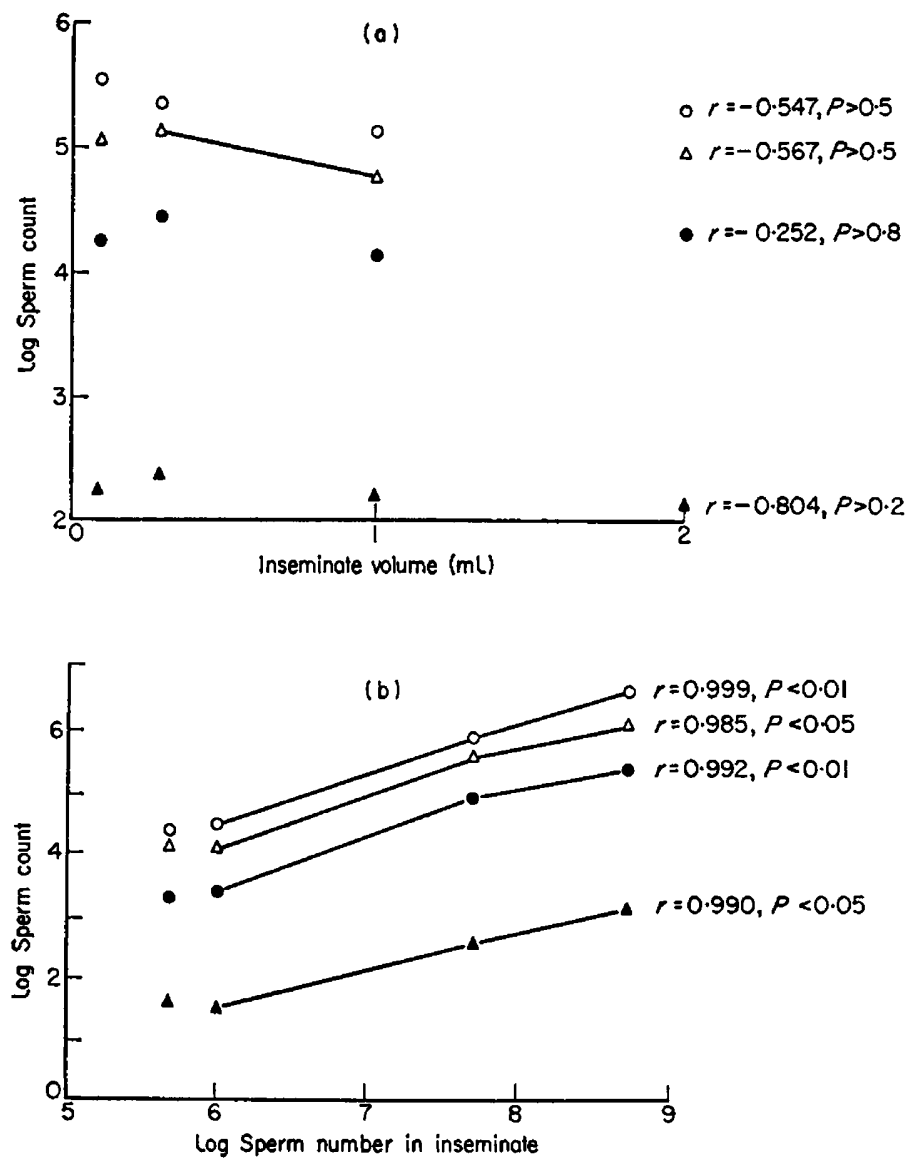

TEXT-FIG. 1. Relationship between regional sperm count in the reproductive tract of inseminated does and (a) the volume of the inseminate, (b) the number of spermatozoa in the inseminate. $O$, Vagina; $\Delta$, cervix; $\bullet$, uterus; $\Delta$, oviduct. Correlation coefficients and probabilities are given. Points connected by a solid line are significantly different from each other $(P<0.05)$.

was little difference between using $0.5 \times 10^{6}$ and $1.0 \times 10^{6}$ spermatozoa, but there was a marked effect with the larger sperm numbers. Statistical analysis of the correlation between regional counts and sperm number in the inseminate showed no significant deviation from linearity $(0.5>P>0 \cdot 1)$.

Correlations between sperm counts in the four areas of the genital tract were evaluated (Text-fig. 2 and Table 3 ) and all regional counts, whether in 

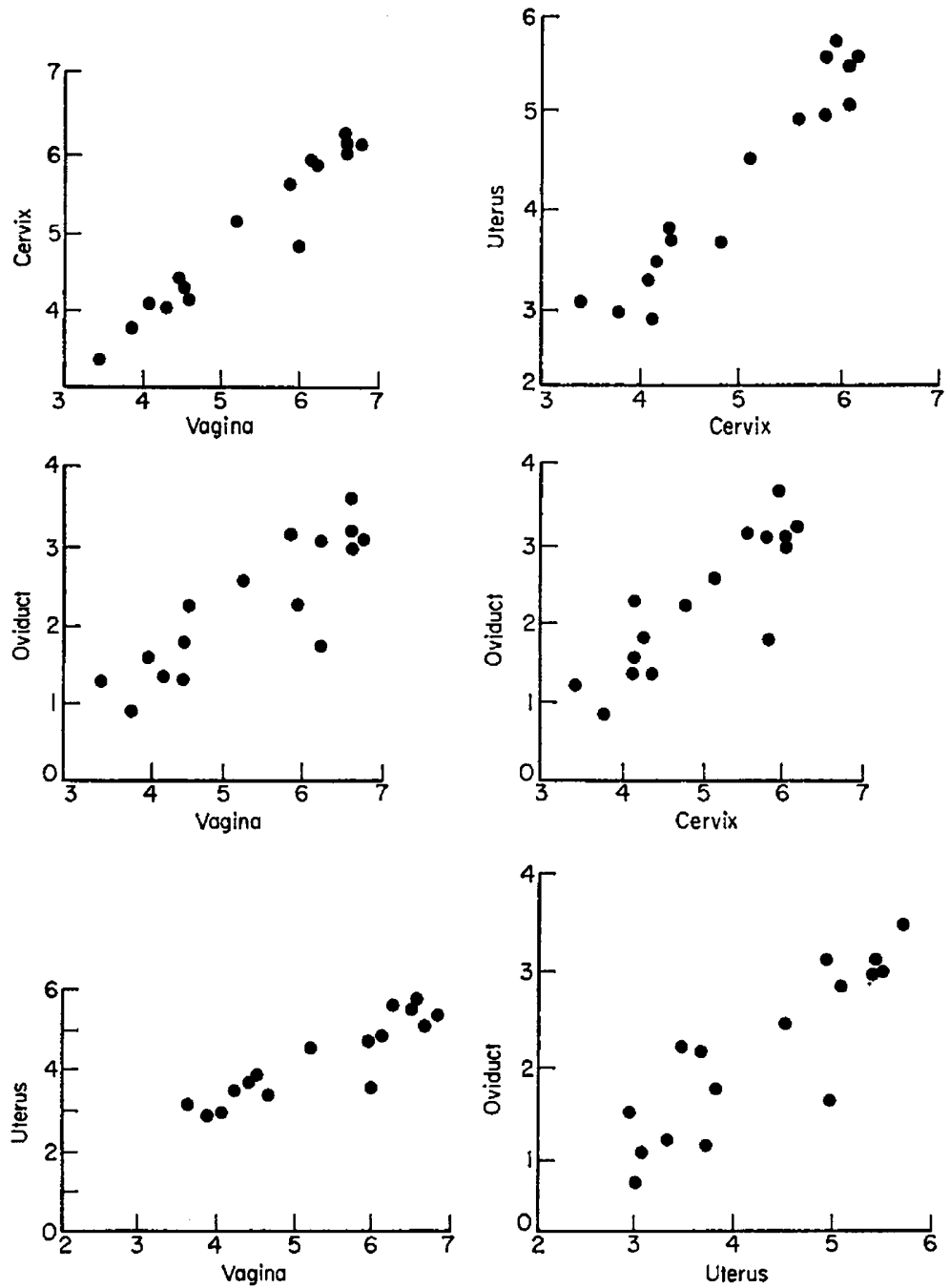

TEXT-FIG. 2. Relationships between the logarithm of sperm counts in various regions of the genital tract of female rabbits $10 \mathrm{hr}$ after insemination.

Table 3. Correlations between the logarithm of the sperm counts from various regions of the reproductive tract of does

\begin{tabular}{l|c|c|c}
\hline $\begin{array}{c}\text { Region of } \\
\text { tract }\end{array}$ & $\begin{array}{c}\text { Correlation } \\
\text { coefficient }(\mathrm{r})\end{array}$ & $\begin{array}{c}\text { Regression } \\
\text { coefficient }(b)\end{array}$ & $\begin{array}{c}\text { Intercept } \\
(a)\end{array}$ \\
\hline Vagina-cervix & $0.907 * * *$ & $0.717( \pm 0 \cdot 105)$ & $+1 \cdot 110$ \\
Vagina-uterus & $0.766^{* * *}$ & $0.653( \pm 0.173)$ & +0.860 \\
Vagina-oviduct & $0.508 * * *$ & $0.447( \pm 0.240)$ & -0.104 \\
Gervix-uterus & $0.715^{* * *}$ & $0.770( \pm 0.238)$ & +0.561 \\
Gervix-oviduct & $0.667 * * *$ & $0.743( \pm 0.262)$ & -1.400 \\
Uterus-oviduct & $0.668^{* * *}$ & $0.691( \pm 0.243)$ & -0.721 \\
\hline
\end{tabular}

Prediction equation $\log y=a+b \log x$ or $y=10^{a} \cdot x^{b}$. Deviation from linear regression $F=0.37$ with 39 degrees of freedom. Regression coefficients are within $95 \%$ confidence limits. 
adjacent areas of the tract or not, appeared to be closely correlated with each other.

Survival of spermatozoa was measured by expressing the total number of spermatozoa recovered from the entire tract as a percentage of the total number in the inseminate. Variation was marked but, taken as a whole, proportionately fewer spermatozoa were recovered when the inseminate contained large numbers, and this was independent of inseminate volume (see Table 4).

Table 4. Analysis of variance relating qualities of the inseminate to survival of rabbit spermatozoa

\begin{tabular}{l|c|l}
\hline \multicolumn{1}{c|}{ Source of variation } & d.f. & \multicolumn{1}{c}{ F } \\
\hline Sperm number & 3 & $6 \cdot 68^{* * *}$ \\
Inseminate volume & 3 & $1 \cdot 62$ \\
Interaction & 9 & $2 \cdot 73^{*}$ \\
$\quad$ Volume $\times$ sperm no. & 27 & \\
$\quad$ Error & $67 \cdot 4$ & \\
Coefficient of variation $(\%)$ & & \\
\hline
\end{tabular}

* $P<0.05 ; * * * P<0.001$.

\section{DISCUSSION}

Overall, the results of this experiment indicate that the number of spermatozoa recovered from any level of the genital tract is a direct function of the total number of spermatozoa in the inseminate, rather than the volume of the inseminate. The importance of sperm number has been stressed previously by Wales et al. (1965) and Wales \& O'Shea (1968) when they measured its effect on live births and egg cleavage respectively. Reduction in the number of eggs fertilized due to the use of very small numbers of spermatozoa has been shown on other occasions (Chang, 1946; Cheng \& Casida, 1948; Bennett \& Vickery, 1969) and the present experiment suggests that the cause of this lowered fertility is due to insufficient spermatozoa at the site of fertilization.

Experiments to determine the minimum number of spermatozoa required for normal fertilization rates have shown variable results and, although between 5000 and 10,000 spermatozoa are usually required, maximum fertility has been achieved when only 500 capacitated spermatozoa were placed in the oviduct (Overstreet, 1970). Cheng \& Casida (1948) and Bennett \& Vickery (1969), on the other hand, claim to have achieved a $21 \%$ and $74 \%$ fertilization rate, respectively, in does inseminated into the vagina with only 16,000 spermatozoa. From the prediction equations of the regression coefficients calculated in this study (in ovariectomized oestrogen-treated does), about sixty spermatozoa would have been in the oviduct at the time of ovulation after intravaginal insemination with 16,000 spermatozoa. Although this number of oviducal spermatozoa is very small, it is worth noting that sperm transport may be considered to be a dynamic process with spermatozoa continually passing through the female tract and so many more spermatozoa are likely to pass through the oviduct during the period of ovulation and egg viability. 
The volume of the inseminate did not appear to influence the number of spermatozoa recovered from the oviduct (see Table 2) and this confirms the results of Wales et al. (1965) who found no correlation between inseminate volume and fertility. Chang (1946), however, found that small inseminate volumes resulted in higher fertilization rates when the number of spermatozoa in the inseminate was kept constant. He suggested that the cervix could accommodate only small volumes of fluid and consequently denser inseminates resulted in more spermatozoa ascending the tract. The results of the present investigation largely lend support to this hypothesis since the analysis of variance (Table 2) indicates that small inseminate volumes result in greater cervical counts than do large volumes. As the cervical lumen is filled with mucus, however, it is unlikely that a small volume of semen per se will be simply taken up by the cervix. It is more likely that with semen of high sperm density, more spermatozoa have the opportunity of migrating into the cervical mucus at the semen/mucus interface than with semen of low sperm density. In consequence, the cervical count will be larger. As close correlations were shown to exist between the number of spermatozoa in the vagina, cervix, uterus and oviduct (Text-fig. 2 and Table 3), it must follow that when more spermatozoa enter the cervix increased numbers will be found in the uterus and oviduct at the time of ovulation. Thus the density of spermatozoa in the inseminate is important in establishing an adequate cervical sperm reservoir.

Chang (1951) found a significant reduction in the number of spermatozoa recovered from the oviduct when the number in the inseminate was reduced to $1.0 \times 10^{6}$ compared with natural mating; he found no reduction when $20 \times 10^{6}$ spermatozoa were used. Conversely, in rabbits mated several times instead of once only, no significant increase in the tubal count was observed (Chang, 1951; Braden, 1953; Morton \& Glover, 1974). These results are surprising, as in this study a linear relationship was found between oviducal count and sperm number in the inseminate (Text-fig. 1). The variance between these results may well be due to a number of different factors such as expulsion of semen due to repeated matings, parity of does and semen quality. Increasing the number of spermatozoa in the inseminate, however, does not result in an increase in the number of spermatozoa in the oviduct of the same proportion and this must reflect the efficiency of the 'barriers' to sperm progression.

The present results show that sperm recovery was proportionately less when does were inseminated with large rather than small numbers of spermatozoa (Table 4). This loss may be due to phagocytosis within the genital tract, expulsion through the vagina, enzymic dissolution or migration into the abdominal cavity. Compared to the number in the inseminate, relatively few spermatozoa are transported through the cervix and so it is unlikely that the deficit can be accounted for either by massive phagocytosis in the uterus and oviduct or by mass migration through the tract into the abdominal cavity. In the present study, many rabbits were seen to void part of the inseminate shortly after insemination, but this loss was not associated with the larger inseminate volumes. It would appear, therefore, that phagocytosis and dissolution of spermatozoa within the vagina are the more likely reasons for the low recovery rate. 


\section{AGKNOWLEDGMENTS}

The authors wish to express their thanks to the Agricultural Research Council and the Population Council for financial support and to Mrs A. Morrison for typing the manuscript.

\section{REFERENGES}

Austrs, G. R. (1948) Number of sperms required for fertilization. Nature, Lond. 162, 534.

BeNNETT, J. P. \& Vickery, B. H. (1969) Hormonal influence of the cervical factor in relation to fertility. 7. Reprod. Med. 3, 123.

Braden, A. W. H. (1953) Distribution of sperms in the genital tract of the female rabbit after coitus. Aust. F. biol. Sci. 6, 693.

Ghang, M. G. (1946) Effect of dilution on fertilizing capacity of rabbit spermatozoa. Science, N.r. 104,361 .

Chang, M. G. (1951) Fertilization in relation to the number of spermatozoa in the fallopian tubes of rabbits. Annali Ostet. Ginec. 73, 918.

Gheng, P. \& Gasida, L. E. (1948) Fertility in the rabbit as affected by the dilution of semen and the number of spermatozoa. Proc. Soc. exp. Biol. Med. 69, 36.

Morton, D. B. (1970) On the transport of spermatozoa in the female rabbit. Ph.D. thesis, University of Liverpool.

Morton, D. B. \& Glover, T. D. (1974) Sperm transport in the female rabbit: the rôle of the cervix. F. Reprod. Fert. 38, 131.

Noyes, R. W., Adams, C. E. \& Walton, A. (1959) The passage of spermatozoa through the genital tract of female rabbits after ovariectomy and oestrogen treatment. $\mathcal{F}$. Endocr. 18, 165.

Overstreet, J. W. (1970) Sperm numbers and fertilization in the rabbit. $\mathcal{J}$. Reprod. Fert. 21, 279.

Rowlands, I. W. (1944) Capacity of hyaluronidase to increase the fertilising power of sperm. Nature, Lond. 154, 332.

Wales, R. G., MARTiN, L. \& O'SheA, T. (1965) Effect of dilution rate and of the number of spermatozoa inseminated on the fertility of rabbits ovulated with chorionic gonadotrophin. F. Reprod. Fert. $10,69$.

Wales, R. G. \& O'SheA, T. (1968) Fertility in rabbits inseminated with diluted and washed spermatozoa. Aust. F. biol. Sci. 21, 181.

Walton, A. (1927) The relation between density of sperm suspension and fertility as determined by artificial insemination of rabbits. Proc. R. Soc. B, 101, 303. 